\title{
CONTINUOUS DEPENDENCE \\ OF LEAST SQUARES SOLUTIONS \\ OF LINEAR BOUNDARY VALUE PROBLEMS
}

\author{
R. KANNAN AND JOHN LOCKER
}

\begin{abstract}
Let $u_{\lambda}$ be the unique least squares solution of minimal norm of the linear boundary value problem $L u-\lambda u=f$, where $L$ is a selfadjoint differential operator in $L^{2}[a, b]$. Working in the Sobolev space $H^{n}[a, b]$, the alternative method is used to examine the continuous dependence of the $u_{\lambda}$ on the parameter $\lambda$ as $\lambda \rightarrow \lambda_{0}$, and the convergent and divergent cases are both characterized.
\end{abstract}

1. Introduction. Let $S$ be the real Hilbert space $L^{2}[a, b]$ with the standard inner product $(u, v)$ and norm $\|u\|$, and let $H^{n}[a, b]$ be the subspace consisting of all functions $u(t)$ in $C^{n-1}[a, b]$ with $u^{(n-1)}$ absolutely continuous on $[a, b]$ and $u^{(n)}$ in $S$. This subspace becomes a Banach space under the norm

$$
|u|_{n}=\sum_{i=0}^{n-1} \max _{a<t<b}\left|u^{(i)}(t)\right|+\left\|u^{(n)}\right\|, \quad u \in H^{n}[a, b] .
$$

We refer to the topology induced by this norm as the $H^{n}$-topology and denote convergence by $u_{i} \rightarrow^{H^{n}} u$.

Let

$$
\tau=\sum_{i=0}^{n} a_{i}(t)\left(\frac{d}{d t}\right)^{i}
$$

be an $n$th order formal differential operator with coefficients $a_{i}(t)$ in $C^{\infty}[a, b]$ and $a_{n}(t) \neq 0$ on $[a, b]$, let

$$
B_{i}(u)=\sum_{j=0}^{n-1} \alpha_{i j} u^{(j)}(a)+\sum_{j=0}^{n-1} \beta_{i j} u^{(j)}(b), \quad i=1, \ldots, n,
$$

be a set of linearly independent boundary values, and let $L$ be the differential operator in $S$ defined by

$$
\mathscr{D}(L)=\left\{u \in H^{n}[a, b] \mid B_{i}(u)=0, i=1, \ldots, n\right\}, \quad L u=\tau u .
$$

We assume that $L$ is selfadjoint, and let $\phi_{i}, i=1,2, \ldots$, be an orthonormal basis for $S$ made up of eigenfunctions of $L$ with corresponding eigenvalues $\lambda_{i}$, $i=1,2, \ldots$, so that $L \phi_{i}=\lambda_{i} \phi_{i}$ and $\left|\lambda_{i}\right| \rightarrow \infty$.

Received by the editors September 18, 1975.

AMS (MOS) subject classifications (1970). Primary 34B05; Secondary 47E05.

Key words and phrases. Continuous dependence, least squares solution, boundary value problem, generalized inverse, alternative method. 
For a fixed function $f \in S$ and a real number $\lambda$, we consider the linear boundary value problem

$$
L u-\lambda u=f .
$$

If we let $L_{\lambda}=L-\lambda I$ and let $P_{\lambda}$ be the orthogonal projection of $S$ onto the null space $\Re\left(L_{\lambda}\right)$, then $I-P_{\lambda}$ is the orthogonal projection of $S$ onto the range $R\left(L_{\lambda}\right)=\Re\left(L_{\lambda}\right)^{\perp}$, and the following well-known theorem characterizes the solvability of equation (1).

THEOREM 1. The boundary value problem (1) is solvable iff $P_{\lambda} f=0$. Thus, if $\lambda \neq \lambda_{i}$ for $i=1,2, \ldots$, then (1) is uniquely solvable, while if $\lambda=\lambda_{i}$ for some $i \geqslant 1$, then (1) either has no solutions or it has infinitely many solutions.

In this paper we are interested in studying least squares solutions of equation (1), and in particular, in determining their dependence on the parameter $\lambda$. Let

$$
H_{\lambda}=\left[L_{\lambda} \mid \mathscr{D}\left(L_{\lambda}\right) \cap \mathscr{N}\left(L_{\lambda}\right)^{\perp}\right]^{-1},
$$

and let $K_{\lambda}=H_{\lambda}\left(I-P_{\lambda}\right)$. The linear operator $K_{\lambda}: S \rightarrow S$ is the generalized inverse of $L_{\lambda}$, and the function $u_{\lambda}=K_{\lambda} f$ is the unique least squares solution of equation (1) of minimal $L^{2}$-norm. In case equation (1) is solvable, $u_{\lambda}$ is an actual solution. These ideas have been discussed in [3].

For a fixed real number $\lambda_{0}$ we ask if $u_{\lambda} \rightarrow{ }^{H^{n}} u_{\lambda_{0}}$ as $\lambda \rightarrow \lambda_{0}$ ? We certainly would expect this to be true if $\lambda_{0}$ is not an eigenvalue of $L$, while the situation when $\lambda_{0}$ is an eigenvalue seems more obscure. In the next section we utilize the alternative method (see [1] or [2]) to characterize when the $u_{\lambda}$ are $H^{n}$-convergent to $u_{\lambda_{0}}$, at the same time obtaining error estimates, and for the divergent case we describe the manner in which the divergence occurs.

2. Continuous dependence. Fixing the real number $\lambda_{0}$, we set

$$
\delta=\inf _{\lambda_{i} \neq \lambda_{0}}\left|\lambda_{i}-\lambda_{0}\right|>0
$$

For $i=1,2, \ldots$ we observe that $L_{\lambda_{0}} \phi_{i}=0$ and $K_{\lambda_{0}} \phi_{i}=0$ for $\lambda_{i}=\lambda_{0}$, and $L_{\lambda_{0}} \phi_{i}=\left(\lambda_{i}-\lambda_{0}\right) \phi_{i}$ and $K_{\lambda_{0}} \phi_{i}=\left(\lambda_{i}-\lambda_{0}\right)^{-1} \phi_{i}$ for $\lambda_{i} \neq \lambda_{0}$, and hence, $K_{\lambda_{0}}$ is a diagonal operator on $S$ with $\left\|K_{\lambda_{0}}\right\|=1 / \delta$. Also, since $K_{\lambda_{0}}$ is continuous from $S$ under the $L^{2}$-topology to $H^{n}[a, b]$ under the $H^{n}$-topology (see [3, p. 180]), we can choose a constant $\gamma>0$ depending on $\lambda_{0}$ such that

$$
\left|K_{\lambda_{0}} u\right|_{n} \leqslant \gamma\|u\| \text { for all } u \in S \text {. }
$$

For each real number $\lambda$ let $u_{\lambda}=v_{\lambda}+w_{\lambda}$ where $v_{\lambda} \in \mathcal{R}\left(L_{\lambda_{0}}\right)$ and $w_{\lambda} \in$ $\Re\left(L_{\lambda_{0}}\right)^{\perp}=\Re\left(L_{\lambda_{0}}\right)$. We know that for $\lambda$ satisfying $0<\left|\lambda-\lambda_{0}\right|<\delta$, the function $u_{\lambda}$ is the unique solution of equation (1), and hence,

$$
L_{\lambda_{0}} u_{\lambda}+\left(\lambda_{0}-\lambda\right) u_{\lambda}-f=0 \text {. }
$$

By the alternative method it follows that for $0<\left|\lambda-\lambda_{0}\right|<\delta$ the functions $u_{\lambda}$ and $v_{\lambda}$ satisfy the equivalent pair of equations

$$
\begin{gathered}
u_{\lambda}+K_{\lambda_{0}}\left[\left(\lambda_{0}-\lambda\right) u_{\lambda}-f\right]=v_{\lambda} \\
P_{\lambda_{0}}\left[\left(\lambda_{0}-\lambda\right) u_{\lambda}-f\right]=0
\end{gathered}
$$


THEOREM 2. The boundary value problem (1) is solvable for $\lambda=\lambda_{0}$ iff the norms $\left\|u_{\lambda}\right\|$ remain bounded as $\lambda \rightarrow \lambda_{0}$, in which case $v_{\lambda}=0$ and $u_{\lambda}=w_{\lambda}$ for $\left|\lambda-\lambda_{0}\right|<\delta$, and $u_{\lambda} \rightarrow^{H^{n}} u_{\lambda_{0}}$ as $\lambda \rightarrow \lambda_{0}$ with the error estimate

$$
\left|u_{\lambda}-u_{\lambda_{0}}\right|_{n} \leqslant(2 \gamma / \delta)\|f\|\left|\lambda-\lambda_{0}\right| \text { for all }\left|\lambda-\lambda_{0}\right|<\delta / 2 \text {. }
$$

Proof. First, assume (1) is solvable for $\lambda=\lambda_{0}$, so $P_{\lambda_{0}} f=0$ by Theorem 1 . From equation (5) we obtain

$$
0=\left(\lambda_{0}-\lambda\right) P_{\lambda_{0}} u_{\lambda}=\left(\lambda_{0}-\lambda\right) v_{\lambda},
$$

and hence, $v_{\lambda}=0$ for $0<\left|\lambda-\lambda_{0}\right|<\delta$. Since $u_{\lambda_{0}}=K_{\lambda_{0}} f$, we get immediately that $v_{\lambda_{0}}=P_{\lambda_{0}} u_{\lambda_{0}}=0$. If $\left|\lambda-\lambda_{0}\right|<\delta / 2$, then from (4) we have

$$
\left\|u_{\lambda}\right\| \leqslant(1 / \delta)(\delta / 2)\left\|u_{\lambda}\right\|+(1 / \delta)\|f\|
$$

or

$$
\left\|u_{\lambda}\right\| \leqslant(2 / \delta)\|f\| \text { for all }\left|\lambda-\lambda_{0}\right|<\delta / 2
$$

Therefore, the norms $\left\|u_{\lambda}\right\|$ remain bounded as $\lambda \rightarrow \lambda_{0}$.

Second, assume the norms $\left\|u_{\lambda}\right\|$ are bounded as $\lambda \rightarrow \lambda_{0}$. From (5) we have

$$
\left\|P_{\lambda_{0}} f\right\| \leqslant\left|\lambda-\lambda_{0}\right|\left\|u_{\lambda}\right\|,
$$

and letting $\lambda \rightarrow \lambda_{0}$ we conclude that $P_{\lambda_{0}} f=0$. By Theorem 1, equation (1) is solvable for $\lambda=\lambda_{0}$. To obtain the error estimate we simply note that (4) can be rewritten as

$$
u_{\lambda}-u_{\lambda_{0}}=\left(\lambda-\lambda_{0}\right) K_{\lambda_{0}} u_{\lambda}
$$

and now apply (2) and (7). This completes the proof.

COROllary. If $\lambda_{0}$ is not an eigenvalue of $L$, then for all $\lambda$ satisfying $\left|\lambda-\lambda_{0}\right|<\delta$ the boundary value problem (1) has $u_{\lambda}$ as its unique solution. Moreover, $u_{\lambda} \rightarrow^{H^{n}} u_{\lambda_{0}}$ as $\lambda \rightarrow \lambda_{0}$, with the rate of convergence determined by equation (6).

THEOREM 3. If the boundary value problem (1) is not solvable for $\lambda=\lambda_{0}$, then

$$
\left\|v_{\lambda}\right\|=\left|\lambda-\lambda_{0}\right|^{-1}\left\|P_{\lambda_{0}} f\right\| \rightarrow \infty \text { as } \lambda \rightarrow \lambda_{0},
$$

$\left\|u_{\lambda}\right\| \rightarrow \infty$ as $\lambda \rightarrow \lambda_{0}$, and $w_{\lambda} \rightarrow{ }^{H^{n}} u_{\lambda_{0}}=w_{\lambda_{0}}$ as $\lambda \rightarrow \lambda_{0}$ with the error estimate

$$
\left|w_{\lambda}-u_{\lambda_{0}}\right|_{n} \leqslant(2 \gamma / \delta)\|f\|\left|\lambda-\lambda_{0}\right| \text { for all }\left|\lambda-\lambda_{0}\right|<\delta / 2 \text {. }
$$

Proof. We obtain (8) from (5) and Theorem 1 , and since $\left\|v_{\lambda}\right\| \leqslant\left\|u_{\lambda}\right\|$, it follows that $\left\|u_{\lambda}\right\| \rightarrow \infty$ as $\lambda \rightarrow \lambda_{0}$. Now $K_{\lambda_{0}} u_{\lambda}=K_{\lambda_{0}} w_{\lambda}$, and hence, we can rewrite (4) as

$$
w_{\lambda}+K_{\lambda_{0}}\left[\left(\lambda_{0}-\lambda\right) w_{\lambda}-f\right]=0
$$

for $\left|\lambda-\lambda_{0}\right|<\delta$. Proceeding as in Theorem 2 we get the bound

$$
\left\|w_{\lambda}\right\| \leqslant(2 / \delta)\|f\| \text { for all }\left|\lambda-\lambda_{0}\right|<\delta / 2,
$$

and a combination of (10) and (11) yields the estimate (9). 


\section{REFERENCES}

1. L. Cesari, Nonlinear analysis, Lecture Notes, C. I. M. E., Bressanone, 1972.

2. J. K. Hale, Applications of alternative problems, Lecture Notes, Brown University, Providence, Rhode Island, 1971.

3. J. Locker, On constructing least squares solutions to two-point boundary value problems, Trans. Amer. Math. Soc. 203 (1975), 175-183.

Department of Mathematical Sciences, University of Missouri at St. Louis, St. Louis, MISSOURI 63121

Department of Mathematics, Colorado State University, Fort Collins, Colorado 80523 